OKHEP-01-02

\title{
THE ADLER FUNCTION FOR LIGHT QUARKS IN ANALYTIC PERTURBATION THEORY
}

\author{
K.A. Milton* \\ Department of Physics and Astronomy, University of Oklahoma, Norman, OK 73019 USA \\ I.L. Solovtsov ${ }^{\dagger}$ and O.P. Solovtsova ${ }^{\ddagger}$ \\ Bogoliubov Laboratory of Theoretical Physics, Joint Institute for Nuclear Research, 141980 \\ Dubna, Moscow Region, Russia
}

(November 13, 2018)

\begin{abstract}
The method of analytic perturbation theory, which avoids the problem of ghost-pole type singularities and gives a self-consistent description of both spacelike and timelike regions, is applied to describe the "light" Adler function corresponding to the non-strange vector channel of the inclusive decay of the $\tau$ lepton. The role of threshold effects is investigated. The behavior of the quark-antiquark system near threshold is described by using a new relativistic resummation factor. It is shown that the method proposed leads to good agreement with the "experimental" Adler function down to the lowest energy scale.
\end{abstract}

11.10.Hi, 12.38.Cy, 13.35.Dx, 14.65.Bt

*E-mail: milton@mail.nhn.ou.edu

${ }^{\dagger}$ E-mail: solovtso@thsun1.jinr.ru

‡E-mail: olsol@thsun1.jinr.ru 


\section{INTRODUCTION}

In studying the relationship between theoretical predictions and experimental data, it is important to connect measured quantities with the most elementary theoretical objects to check direct consequences of the theory without essential use of model assumptions. Some single-argument functions which are directly connected with experimentally measured quantities can play the role of these objects. A theoretical description of inclusive processes can be made in terms of functions of this sort. Among them is the Adler function [1] which can be extracted from the experimental data for the process of $e^{+} e^{-}$annihilation into hadrons and the inclusive decay of the $\tau$ lepton. The mass of the $\tau$ lepton, $M_{\tau}=1.777 \mathrm{GeV}$, is large enough in order to produce decays with a hadronic mode. At the same time, in the context of QCD, the mass is sufficiently small to allow one to investigate effects lying beyond the framework of the perturbative approach. At present, there is rich experimental material obtained from hadronic decays of the $\tau$ lepton. The first theoretical analysis of hadronic decays of a heavy lepton was performed in 1971 [2] well before the experimental discovery of the $\tau$-lepton in 1975. Since then, the properties of the $\tau$ have been studied very intensively.

The ratio of hadronic to leptonic widths for the inclusive decay of the $\tau$-lepton,

$$
R_{\tau}=\frac{\Gamma\left(\tau^{-} \rightarrow \text { hadrons } \nu_{\tau}\right)}{\Gamma\left(\tau^{-} \rightarrow \ell \bar{\nu}_{\ell} \nu_{\tau}\right)}
$$

is the most precise one for extracting of the values of the fundamental QCD parameters at a low energy scale [3]. The initial theoretical expression for $R_{\tau}$ contains an integral over timelike momentum

$$
R_{\tau}=\frac{2}{\pi} \int_{0}^{M_{\tau}^{2}} \frac{d s}{M_{\tau}^{2}}\left(1-\frac{s}{M_{\tau}^{2}}\right)^{2}\left(1+2 \frac{s}{M_{\tau}^{2}}\right) \operatorname{Im} \Pi(s),
$$

which extends down to small $s$ and cannot be directly calculated in the framework of standard perturbation theory $(\mathrm{PT})$. Indeed, the hadronic correlation function $\Pi(s)$ is parametrized by the perturbative running coupling which has unphysical singularities and, therefore, is ill-defined in the region of small momenta. To avoid this problem, one usually applies the following procedure. The initial integral (四) is rewritten by using the Cauchy theorem in the form of a contour integral in the complex plane with the contour running around a circle with radius $M_{\tau}^{2}$ [4. 5 ]:

$$
R_{\tau}=\frac{1}{2 \pi \mathrm{i}} \oint_{|z|=M_{\tau}^{2}} \frac{d z}{z}\left(1-\frac{z}{M_{\tau}^{2}}\right)^{3}\left(1+\frac{z}{M_{\tau}^{2}}\right) D(z)
$$

where $D(z)=-z d \Pi(z) / d z$ is the Adler function. This trick allows one, in principle, to avoid the problem of a direct calculation of the $R_{\tau}$ ratio by Eq. (11). However, in order to perform this transformation self-consistently, it is necessary to maintain correct analytic properties of the hadronic correlation function, which are violated in the framework of standard PT. The analytic approach to QCD [6], the so-called analytic perturbation theory (APT) [7,8], maintains needed analytic properties and allows one to give meaning to the initial expression. The APT description can be equivalently phrased either on the basis of the expression (1) or 
on the contour representation (2) [7]. The information obtained in $\tau$ measurements allows one to construct various "experimental" curves. In particular, in paper [9] a quantity $R_{\tau}\left(s_{0}\right)$ with a variable "mass" $s_{0} \leq M_{\tau}^{2}$, has been considered. This quantity, defined for timelike momenta, is similar to the "smeared" functions constructed, for example, according to the Poggio-Quinn-Weinberg method [10]. The $\tau$-decay data allow us also to determine quantities in the Euclidean region, including the Adler function. This function can be extracted from the $\tau$ data down to the lowest energy scale [11].

In this paper we study the Adler $D$-function corresponding to $\tau$ decay mediated by the non-strange vector current via $W^{-} \rightarrow d \bar{u}$. We use the APT method which does not encounter the problem of unphysical singularities of the running coupling and gives a self-consistent description of both the timelike, Eq. (1), and the spacelike, Eq. (2), regions.

The region of integration in Eq. (1) includes the vicinity of the quark-antiquark threshold. The perturbative expansion breaks down in this neighborhood due to singularities at $s=$ $\left(m_{q}+m_{\bar{q}}\right)^{2}$ [12,10]. Thus any finite order of the perturbative expansion is unreliable near quark thresholds and, therefore, all singular terms of the $\left(\alpha_{S} / v\right)^{n}$ type, where $v$ is the relative velocity of the quarks, have to be summed. Note that this problem cannot be avoided by using the contour representation (2) instead of Eq. (1), because these expressions should be equivalent to each other in the framework of a consistent method. For heavy quark systems one usually uses the nonrelativistic resummation factor obtained by using the Schrödinger equation with the Coulomb potential, which is known as the SommerfeldSakharov factor [13,14]. But for a systematic description of the threshold region in the system of light quarks it is necessary to apply a relativistic approach. Here, we take into account threshold effects by using a new relativistic resummation factor proposed in Ref. [15], which was obtained for a QCD-like potential.

\section{ANALYTIC APPROACH TO $\tau$ DECAY}

We start our consideration with a three-loop PT and APT analysis, neglecting, in the beginning, quark masses. It is convenient to separate the QCD contribution by representing the $R_{\tau}$ ratio in the form

$$
R_{\tau}=R_{\tau}^{0}\left(1+\delta_{\mathrm{QCD}}\right)
$$

where $R_{\tau}^{0}$ corresponds to the parton level description and $\delta_{\mathrm{QCD}}$ is the QCD correction. We introduce QCD contributions to the imaginary part of the hadronic correlator, $r(s)$, and to the corresponding Adler function, $d(z)$ as follows:

$$
\mathcal{R}(s)=\operatorname{Im} \Pi(s+i \epsilon) / \pi R_{\tau}^{0} \propto 1+r, \quad D(z) \propto 1+d(z) .
$$

Then, one can write $\delta_{\mathrm{QCD}}$ as an integral over timelike momentum (Minkowskian region)

$$
\delta_{\mathrm{QCD}}=2 \int_{0}^{M_{\tau}^{2}} \frac{d s}{M_{\tau}^{2}}\left(1-\frac{s}{M_{\tau}^{2}}\right)^{2}\left(1+2 \frac{s}{M_{\tau}^{2}}\right) r(s),
$$

or as a contour integral in the complex plane (Euclidean region) 


$$
\delta_{\mathrm{QCD}}=\frac{1}{2 \pi i} \oint_{|z|=M_{\tau}^{2}} \frac{d z}{z}\left(1-\frac{z}{M_{\tau}^{2}}\right)^{3}\left(1+\frac{z}{M_{\tau}^{2}}\right) d(z)
$$

We now consider $\delta_{\mathrm{QCD}}$ in the framework the PT and APT methods.

\section{A. Perturbation theory}

The PT description is based on the contour representation and can be developed in the following two ways. In Braaten's (Br) method [4] the quantity (5) is represented in the form of truncated power series with the expansion parameter $a_{\tau} \equiv \alpha_{S}\left(M_{\tau}^{2}\right) / \pi$. In this case the three-loop representation for $\delta_{\mathrm{QCD}}$ is

$$
\delta_{\mathrm{QCD}}^{\mathrm{Br}}=a_{\tau}+r_{1} a_{\tau}^{2}+r_{2} a_{\tau}^{3}
$$

where the coefficients $r_{1}$ and $r_{2}$ in the $\overline{\mathrm{MS}}$ scheme with three active flavors are $r_{1}=5.2023$ and $r_{2}=26.366$ 所.

The method proposed by Le Diberder and Pich (LP) [5] uses the PT expansion of the $d$-function

$$
d(z)=a(z)+d_{1} a^{2}(z)+d_{2} a^{3}(z),
$$

where in the $\overline{\mathrm{MS}}$-scheme $d_{1}=1.640$ and $d_{2}=6.371$ [16 for three active quarks. The threeloop PT running coupling, $a(z)$, is commonly written in the form of an expansion in inverse powers of $L$ [3]. In the $\overline{\mathrm{MS}}$ scheme it is

$$
a(z)=\frac{4}{\beta_{0} L}\left\{1-\frac{\beta_{1}}{\beta_{0}^{2}} \frac{\ln L}{L}+\frac{1}{L^{2}}\left[\frac{\beta_{1}^{2}}{\beta_{0}^{4}}\left(\ln ^{2} L-\ln L-1\right)+\frac{\beta_{2}}{\beta_{0}^{3}}\right]\right\}, L \equiv \ln \left(-z / \Lambda^{2}\right),
$$

where $\beta_{0}=11-2 n_{f} / 3, \beta_{1}=102-38 n_{f} / 3$ and $\beta_{2}^{\overline{\mathrm{MS}}}=2857 / 2-5033 n_{f} / 18+325 n_{f}^{2} / 54$ are the first three $\beta$-function coefficients. The substitution of Eq. (可) into Eq. (5) leads to the following non-power representation

$$
\delta_{\mathrm{QCD}}^{\mathrm{LP}}=A^{(1)}(a)+d_{1} A^{(2)}(a)+d_{2} A^{(2)}(a)
$$

with

$$
A^{(n)}(a)=\frac{1}{2 \pi i} \oint_{|z|=M_{\tau}^{2}} \frac{d z}{z}\left(1-\frac{z}{M_{\tau}^{2}}\right)^{3}\left(1+\frac{z}{M_{\tau}^{2}}\right) a^{n}(z) .
$$

As noted above, transformation to the contour representation (5) requires the existence of certain analytic properties of the correlator: namely, it must be an analytic function in the complex $z$-plane with a cut along the positive real axis. The correlator parametrized, as usual, by the PT running coupling does not have this virtue. Moreover, the conventional renormalization group method determines the running coupling in the spacelike region, whereas the initial expression (11) contains an integration over timelike momentum, and there is the question of how to parametrize a quantity defined for timelike momentum 
transfers [17]. To perform this procedure self-consistently, it is important to maintain correct analytic properties of the hadronic correlator [18 20]. Because of this failure of analyticity, Eqs. (4) and (5) are not equivalent in the framework of PT and, if one remains within PT, it is difficult to estimate the errors introduced by this transformation. However, using the APT method, it is possible to resolve these problems.t]

\section{B. Analytic perturbation theory}

In the framework of the analytic approach, 2 the functions $d(z)$ and $r(s)$ are expressed in terms of the effective spectral function $\rho(\sigma)$ [6, 19]

$$
d(z)=\frac{1}{\pi} \int_{0}^{\infty} \frac{d \sigma}{\sigma-z} \rho(\sigma), \quad r(s)=\frac{1}{\pi} \int_{s}^{\infty} \frac{d \sigma}{\sigma} \rho(\sigma) .
$$

The APT spectral function is defined as the imaginary part of the perturbative approximation to $d_{\mathrm{pt}}$ on the physical cut

$$
\rho(\sigma)=\varrho_{0}(\sigma)+d_{1} \varrho_{1}(\sigma)+d_{2} \varrho_{2}(\sigma)
$$

where

$$
\varrho_{n}(\sigma)=\operatorname{Im}\left[a_{\mathrm{pt}}^{n+1}(\sigma+i \epsilon)\right] .
$$

The function $\varrho_{0}(\sigma)$ in Eq. (12) defines the analytic spacelike, $a_{\text {an }}(z)$, and timelike, $\tilde{a}_{\text {an }}(s)$, running couplings as follows

$$
a_{\mathrm{an}}(z)=\frac{1}{\pi} \int_{0}^{\infty} \frac{d \sigma}{\sigma-z} \varrho_{0}(\sigma), \quad \tilde{a}_{\mathrm{an}}(s)=\frac{1}{\pi} \int_{s}^{\infty} \frac{d \sigma}{\sigma} \varrho_{0}(\sigma)
$$

As has been argued from general principles, the behavior of these couplings cannot be the same [22]. It should be stressed that, unlike the PT running coupling, the analytic running coupling has no unphysical singularities (the ghost pole and branch points) and, therefore, possesses the correct analytic properties, arising from Källén-Lehmann analyticity reflecting the general principles of the theory. For example, the one-loop APT result is [6.19]

$$
a_{\mathrm{an}}^{(1)}(z)=a_{\mathrm{pt}}^{(1)}(z)+\frac{4}{\beta_{0}} \frac{\Lambda^{2}}{\Lambda^{2}+z}, \quad \tilde{a}_{\mathrm{an}}^{(1)}(s)=\frac{4}{\beta_{0}}\left[\frac{1}{2}-\frac{1}{\pi} \arctan \frac{\ln \left(s / \Lambda^{2}\right)}{\pi}\right],
$$

where $a_{\mathrm{pt}}^{(1)}(z)=4 /\left[\beta_{0} \ln \left(-z / \Lambda^{2}\right)\right]$.

The analytic running couplings (the exact two-loop and the three-loop after an approximation) can be written explicitly in the term of the Lambert function [23,24]. However,

\footnotetext{
${ }^{1}$ The nonperturbative $a$-expansion technique in QCD [21] also leads to a well-defined procedure of analytic continuation [18].

${ }^{2}$ To distinguish APT and PT cases, we will use subscripts "an" and "pt".
} 
in the framework of the APT approach there is a little sensitivity to the approximation in solving the renormalization group equation for the running coupling [7, 8]. In the following, we use an explicit form for the analytic running coupling in the timelike region, $\tilde{a}_{\text {an }}(s)$, that is derived by using the formula (8) in Eq. (12). The corresponding expression reads

$$
\tilde{a}_{\mathrm{an}}(s)=\tilde{a}_{\mathrm{an}}^{(1)}(s)+\tilde{\Delta}_{1}(s)+\tilde{\Delta}_{2}(s),
$$

where $\tilde{a}_{\text {an }}^{(1)}(s)$ is given in Eq. (15) and

$$
\begin{aligned}
& \tilde{\Delta}_{1}=-\frac{4 \beta_{1}}{\beta_{0}^{3}} \frac{1}{B^{2}}\left[\ln B+1-A \frac{\ln \left(s / \Lambda^{2}\right)}{\pi}\right], \\
& \tilde{\Delta}_{2}=\frac{4 \pi \beta_{1}^{2}}{\beta_{0}^{5}} \frac{1}{B^{4}}\left\{\left(\frac{\beta_{2} \beta_{0}}{\beta_{1}^{2}}-1-A^{2}+\ln ^{2} B\right) \frac{\ln \left(s / \Lambda^{2}\right)}{\pi}+\left[1-\frac{\ln ^{2}\left(s / \Lambda^{2}\right)}{\pi^{2}}\right] A \ln B\right\}, \\
& A(s)=\frac{\beta_{0}}{4} \pi \tilde{a}_{\text {an }}^{(1)}(s), \quad B(s) \equiv \sqrt{\pi^{2}+\ln ^{2}\left(s / \Lambda^{2}\right)} .
\end{aligned}
$$

For the QCD correction $r(s)$ one can write down [25]

$$
r_{\mathrm{an}}(s)=\tilde{a}_{\mathrm{an}}(s)+\Delta r_{1}(s)+\Delta r_{2}(s),
$$

where $\tilde{a}_{\text {an }}(s)$ is given by Eq. (16) and

$$
\begin{aligned}
& \Delta r_{1}=d_{1}\left(\frac{4}{\beta_{0}}\right)^{2} \frac{1}{B^{2}}\left\{1-\frac{\beta_{1}}{\beta_{0}^{2}} \frac{\pi}{B^{2}}\left[(2 \ln B+1) \frac{\ln \left(s / \Lambda^{2}\right)}{\pi}+A-A \frac{\ln ^{2}\left(s / \Lambda^{2}\right)}{\pi^{2}}\right]\right\}, \\
& \Delta r_{2}=d_{2}\left(\frac{4}{\beta_{0}}\right)^{3} \frac{1}{B^{4}} \ln \left(s / \Lambda^{2}\right) .
\end{aligned}
$$

Using Eq. (4) or equivalently Eq. (5), we obtain the QCD correction to the $R_{\tau}$-ratio in terms of $\rho(\sigma)$ as follows

$$
\delta_{\mathrm{an}}=\frac{1}{\pi} \int_{M_{\tau}^{2}}^{\infty} \frac{d \sigma}{\sigma} \rho(\sigma)+\frac{1}{\pi} \int_{0}^{M_{\tau}^{2}} \frac{d \sigma}{\sigma}\left[2 \frac{\sigma}{M_{\tau}^{2}}-2\left(\frac{\sigma}{M_{\tau}^{2}}\right)^{3}+\left(\frac{\sigma}{M_{\tau}^{2}}\right)^{4}\right] \rho(\sigma) .
$$

It is obvious that the first term of this expression is $r_{\text {an }}$ evaluated at the $\tau$ mass and that $\delta_{\mathrm{an}}$ is not representable as a series expansion in the running coupling.

The difference between the PT (LP) and APT contributions to $R_{\tau}$ can be transparently shown by the one-loop relation:

$$
\delta_{\mathrm{an}}^{(1)}=\delta_{\mathrm{pt}}^{(1)}-\frac{8}{\beta_{0}} \frac{\Lambda^{2}}{M_{\tau}^{2}}+\mathrm{O}\left(\Lambda^{4} / M_{\tau}^{4}\right) .
$$

The additional term, which is "invisible" in the perturbative expansion, turns out to be important numerically [7,26]. Due to the negative sign of this term, the QCD scale is larger in this method, $\Lambda_{\mathrm{an}}>\Lambda_{\mathrm{pt}}$, at the same value of the QCD correction: $\delta_{\mathrm{an}}\left(\Lambda_{\mathrm{an}}\right)=\delta_{\mathrm{pt}}\left(\Lambda_{\mathrm{pt}}\right)=\delta^{\exp }$. It should be noted that due to the difference of shapes of the APT and PT running couplings (see, for example, Ref. [27]), their values at the $\tau$ scale do not differ very much [28]. 
The APT analysis of the $\tau$ decay in the three-loop level has been performed in Ref. [28]. This investigation together with other results (see, for example, Refs. [8,29,30]) allows us to formulate the following features of the APT method: (i) this approach maintains the correct analytic properties and leads to a self-consistent procedure of analytic continuation from the spacelike to the timelike region; (ii) it has much improved convergence properties and turns out to be stable with respect to higher-loop corrections; (iii) renormalization scheme dependence of the results obtained within this method is reduced dramatically.

\section{The vector channel in $\tau$ decay}

Experimentally the $R_{\tau}$-ratio can be separated into three parts

$$
R_{\tau}=R_{\tau, V}+R_{\tau, A}+R_{\tau, S}
$$

The terms $R_{\tau, V}$ and $R_{\tau, A}$ are contributions coming from the non-strange hadronic decays associated with vector $(V)$ and axial-vector $(A)$ quark currents respectively, and $R_{\tau, S}$ contains strange decays $(S)$.

Within the perturbative approximation with massless quarks the vector and axial-vector contributions to $R_{\tau}$ coincide with each other

$$
R_{\tau, V}=R_{\tau, A}=\frac{3}{2}\left|V_{u d}\right|^{2}\left(1+\delta_{\mathrm{QCD}}\right),
$$

where $\left|V_{u d}\right|$ denotes the Cabibbo-Kobayashi-Maskawa matrix element. However, the experimental measurements [31.32] show that these components are not equal to each other. The corresponding difference is associated with non-perturbative QCD effects which are usually described in the form of power corrections. The experimental data for the isovector spectral function of the ALEPH Collaboration [31] have been used in Ref. [11] to extract the Adler $D_{V}$-function which we show as the dashed line in Fig. [1. The experimental $D$-function turns out to be a smooth function without any trace of resonance structure. The $D$-function obtained in Ref. [33] from the data for electron-positron annihilation into hadrons also has a similar property. One can expect that the Adler $D$-function defined in the Euclidean region reflects more adequately the quark-hadron duality than do quantities determined in the Minkowskian region, [] and, therefore, is more suitable for relating theoretical predictions with experimental data. It is obvious that the PT approximation cannot be applied at the low energy scale. Besides, any finite order of the operator product expansion fails to describe the infrared tail of the $D$-function. APT's good behavior in the infrared region, in principle, allows us to consider the $D$-function down to a low energy scale.

\footnotetext{
${ }^{3}$ The Minkowskian and Euclidean characteristics which are associated with the process of electronpositron annihilation into hadrons have been considered in Ref. [34].
} 


\section{THE VECTOR CHANNEL D-FUNCTION}

In this section we describe the Adler function corresponding to inclusive $\tau$ decay. By using a dispersion relation for the hadronic correlator $\Pi\left(q^{2}\right)$, one can represent the Adler function as follows

$$
D\left(Q^{2}\right)=Q^{2} \int_{0}^{\infty} d s \frac{\mathcal{R}(s)}{\left(s+Q^{2}\right)^{2}}
$$

We will derive $\mathcal{R}(s)$ by applying the APT approach while incorporating threshold resummation.

\section{A. Threshold effects}

A description of quark-antiquark systems near threshold requires us to take into account the resummation factor which summarizes the threshold singularities of the perturbative series of the $\left(\alpha_{S} / v\right)^{n}$ type. In a nonrelativistic approximation, this is the well known Sommerfeld-Sakharov factor [13,14]. For a systematic relativistic analysis of quark-antiquark systems, it is essential from the very beginning to have a relativistic generalization of this factor. Moreover, it is important to take into account the difference between the Coulomb potential in the case of QED and the quark-antiquark potential in the case of QCD. This QCD relativistic factor has been proposed in Ref. [15] to have the form

$$
S(\chi)=\frac{X(\chi)}{1-\exp [-X(\chi)]}, \quad X(\chi)=\frac{4 \pi \alpha_{S}}{3 \sinh \chi}
$$

where $\chi$ is the rapidity which related to $s$ by $2 m \cosh \chi=\sqrt{s}$. The relativistic resummation factor (25) reproduces both the expected nonrelativistic and ultrarelativistic limits and corresponds to a QCD-like quark-antiquark potential.

A convenient way to incorporate quark mass effects is to use an approximate expression [10,12] which here can be written as

$$
\mathcal{R}(s)=T(v)[1+g(v) r(s)] \Theta\left(s-4 m^{2}\right),
$$

where

$$
T(v)=v \frac{3-v^{2}}{2}, g(v)=\frac{4 \pi}{3}\left[\frac{\pi}{2 v}-\frac{3+v}{4}\left(\frac{\pi}{2}-\frac{3}{4 \pi}\right)\right], v=\sqrt{1-\frac{4 m^{2}}{s}} .
$$

We introduce effective quark masses, which accumulate some non-perturbative contributions and turn out to be close to the constituent masses. In the description of the non-strange vector component of the $D$-function we neglect the difference of the quark mass values and set $m_{u}=m_{d}=m$.

The threshold resummation factor (25) leads to the following modification of the expression $(26)$

$$
\mathcal{R}_{V}(s)=T(v)\left[S(\chi)-\frac{1}{2} X(\chi)+g(v) r(s)\right] \Theta\left(s-4 m^{2}\right)
$$


which one can use to calculate the vector component of the $R_{\tau}$ ratio

$$
R_{\tau, V}=3 S_{\mathrm{EW}}\left|V_{u d}\right|^{2} \int_{0}^{M_{\tau}^{2}} \frac{d s}{M_{\tau}^{2}}\left(1-\frac{s}{M_{\tau}^{2}}\right)^{2}\left(1+\frac{2 s}{M_{\tau}^{2}}\right) \mathcal{R}_{V}(s)
$$

where $S_{\mathrm{EW}}$ denotes the electroweak factor 35 and the light vector $D$-function is defined by Eq. (24) with $\mathcal{R} \rightarrow \mathcal{R}_{V}$.

\section{B. Results}

We derive the $D_{V}$-function and the value of $R_{\tau, V}$ by using Eq. (28) with the expression (18) for $r(s)$ and the timelike running coupling (16) in the $S$-factor. In Fig. I we plot this $D$-function (solid curve) which was calculated by using the parameter $\Lambda=420 \mathrm{MeV}$ and the value of the quark masses $m_{u}=m_{d}=250 \mathrm{MeV}$. Note that, practically, the same values of the light quark masses have been obtained in Refs. 36, 37. These values are close to the constituent quark masses and incorporate some nonperturbative effects. The shape of the infrared tail of the $D$-function is rather sensitive to the value of these masses. We obtain the value of $R_{\tau, V}=1.77$ which agrees well with the experimental data presented by the ALEPH, $R_{\tau, V}^{\text {expt }}=1.775 \pm 0.017$ [31], and the OPAL, $R_{\tau, V}^{\text {expt }}=1.764 \pm 0.016$ [32], collaborations. The value of the parameter $\Lambda$ obtained here is slightly more than in the standard analysis. For example, the ALEPH Collaboration result is $\Lambda=370 \pm 13_{\text {expt }} \pm 38_{\text {theor }} \mathrm{MeV}$. In this connection, note that a more accurate account of the so-called $\pi^{2}$-terms in the treatment of high energy experimental data for timelike processes leads to an increase in the value of $\alpha_{S}$ [20]. It should be emphasized that the value of $\Lambda$ turns out to be significantly smaller than the value extracted from the $\tau$ data in Ref. [28], which shows the importance of threshold effects. [Recall that in Fig. 1 w we also show the experimental curve (dashed line) and the curve corresponding to the perturbative result with power corrections (dotted line) which are taken from Ref. [11].] Note also that using the above values of quark masses, we find that the one-loop APT description gives a result (not shown in the Figure) which is close to the three-loop one and reproduces well the experimental behavior of the $D$-function.

\section{CONCLUSION}

In this paper we have considered the Adler function corresponding to the non-strange vector channel data from $\tau$ decay. This function, defined in the Euclidean region, is a smooth function and represents a convenient testing ground for theoretical methods. We have proposed the method of the "light" vector $D$-function description. The conventional method of approximating this function as a sum of perturbative terms and power corrections cannot describe the low energy scale region because both the logarithmic and power expansions diverge at small momenta. We have used the analytic approach to QCD which is not in conflict with the general principles of the theory and, in the infrared region, has a regular behavior. The new relativistic resummation factor, which corresponds to a QCD-like quark-antiquark quasipotential, has been used to incorporate the threshold effects. We have shown that our approach allows us to describe well the experimental data for $\tau$-lepton decay 
in terms of the $D$-function down to the lowest energy scale and for $R_{\tau}$ in the non-strange vector channel. We have found that the influence of relativistic threshold resummation is important and leads to an significant reduction of the value of the QCD scale parameter $\Lambda$ extracted from the $\tau$ data.

\section{ACKNOWLEDGMENTS}

The authors would like to thank D.V. Shirkov and A.N. Sissakian for interest in this work. Partial support of the work by the US National Science Foundation, grant PHY9600421, and by the US Department of Energy, grant DE-FG-03-98ER41066, and by the RFBR, grants 99-01-00091, 99-02-17727, 00-15-96691 is gratefully acknowledged. The work of ILS and OPS is also supported in part by the University of Oklahoma, through its College of Arts and Science, the Vice President for Research, and the Department of Physics and Astronomy. 


\section{REFERENCES}

[1] S.L. Adler, Phys. Rev. D 10, 3714 (1974).

[2] Y.S. Tsai, Phys. Rev. D 4, 2821 (1971).

[3] Particle Data Group, C. Caso et al., Eur. Phys. J. C 3, 1 (1998); D.E. Groom et al., Eur. Phys. J. C 15, 1 (2000).

[4] E. Braaten, Phys. Rev. Lett. 60, 1606 (1988); Phys. Rev. D 39, 1458 (1989); E. Braaten, S. Narison, and A. Pich, Nucl. Phys. B 373, 581 (1992).

[5] F. Le Diberder and A. Pich, Phys. Lett. B 286, 147 (1992).

[6] D.V. Shirkov and I.L. Solovtsov, JINR Rap. Comm. 2[76], 1996, hep-ph/9604363; Phys. Rev. Lett. 79, 1209 (1997).

[7] K.A. Milton, I.L. Solovtsov, and O.P. Solovtsova, Phys. Lett. B 415, 104 (1997).

[8] K.A. Milton, I.L. Solovtsov, and O.P. Solovtsova, in Proc. of the XXIX Int. Conference on High Energy Physics, Vancouver, B.C. Canada, July 23-29 1998, edited by A. Astbury, D. Axen and J. Robinson (World Scientific, Singapore, 1999) vol. II, p. 1608.

[9] M. Girone and M. Neubert, Phys. Rev. Lett. 76, 3061 (1996).

[10] E.C. Poggio, H.R. Quinn, and S. Weinberg, Phys. Rev. D 13, 1958 (1976).

[11] S. Peris, M. Perrottet, and E. de Rafael, JHEP 05, 011 (1998).

[12] T. Appelquist and H.D. Politzer, Phys. Rev. Lett. 34, 43 (1975); Phys. Rev. D 12, 1404 (1975).

[13] A. Sommerfeld, Atombau und Spektrallinien, vol. II (Vieweg, Braunschweig, 1939).

[14] A.D. Sakharov, Zh. Eksp. Teor. Fiz. 18, 631 (1948).

[15] K. A. Milton and I. L. Solovtsov, OKHEP-00-06, hep-ph/0005175.

[16] S.G. Gorishny, A.L. Kataev, and S.A. Larin, Phys. Lett. B 259, 144 (1991).

[17] M.R. Pennington and G.G Ross, Phys. Lett. 102B, 167 (1981); A.V. Radyushkin, Preprint JINR, E2-82-159 (1982); N.V. Krasnokov and A.A. Pivovarov, Phys. Lett. 116B, 168 (1982).

[18] H.F. Jones and I.L. Solovtsov, Phys. Lett. B 349, 519 (1995); in Proc. of the Int. Europhysics Conference on High Energy Physics, Brussels, Belgium, 27 Jul-2 Aug 1995, edited by J. Lemonne et al. (World Scientific, Singapore, 1996) p. 242.

[19] K.A. Milton and I.L. Solovtsov, Phys. Rev. D 55, 5295 (1997); K.A. Milton and O.P. Solovtsova, Phys. Rev. D 57, 5402 (1998).

[20] D.V. Shirkov, Preprint JINR E2-2000-211, hep-ph/0009106, v. 2; Preprint JINR E22000-298, hep-ph/0012283.

[21] I.L. Solovtsov, Phys. Lett. B 327, 335 (1994); Phys. Lett. B 340, 245 (1994).

[22] K.A. Milton and I.L. Solovtsov, Phys. Rev. D 59, 107701 (1999).

[23] B. Magradze, in Proc. of the Int. Seminar "QUARKs-98", Suzdal, Russia, May 1724, 1998, edited by F.L. Bezrukov et. al. (INR Publ., Moscow, 2000), p. 158, hepph/9808247; Int. J. Mod. Phys. A 15, 2715 (2000); Preprint JINR E2-2000-222, hepph/0010070.

[24] E. Gardi, G. Grunberg, and M. Karliner, JHEP 07, 007 (1998).

[25] B.V. Geshkenbein and B.L. Ioffe, JETP Lett. 70, 161 (1999), hep-ph/9906406.

[26] O.P. Solovtsova, JETP Lett. 64, 714 (1996).

[27] K.A. Milton, I.L. Solovtsov, and O.P. Solovtsova, Phys. Rev. D 60, 016001 (1999). 
[28] K.A. Milton, I.L. Solovtsov, O.P. Solovtsova, and V.I. Yasnov, Eur. Phys. J. C 14, 495 (2000).

[29] I.L. Solovtsov and D.V. Shirkov, Phys. Lett. B 442, 344 (1998); Theor. Math. Phys. 120, 1220 (1999).

[30] D.V. Shirkov, Theor. Math. Phys. 119, 438 (1999), hep-th/9810246.

[31] ALEPH Collaboration, R. Barate et al., Eur. Phys. J. C 4, 409 (1998); Z. Phys. C 76, 15 (1997).

[32] OPAL Collaboration, K. Ackerstaff et al., Eur. Phys. J. C 7, 571 (1999).

[33] S. Eidelman, F. Jegerlehner, A.L. Kataev, and O. Veretin, Phys. Lett. B 454, 369 (1999).

[34] K.A. Milton, I.L. Solovtsov, and O.P. Solovtsova, Eur. Phys. J. C 13, 497 (2000).

[35] W.J. Marciano and A. Sirlin, Phys. Rev. Lett. 56, 22 (1986); 61, 1815 (1988).

[36] A.I. Sanda, Phys. Rev. Lett. 42, 1658 (1979).

[37] I.L. Solovtsov and D.V. Shirkov, talk given at The Int. Workshop on " $e^{+} e^{-}$collisions: from $\phi$ to $J / \Psi "$, Novosibirsk, Russia, March 1-5 1999, hep-ph/9906495. 


\section{FIGURES}

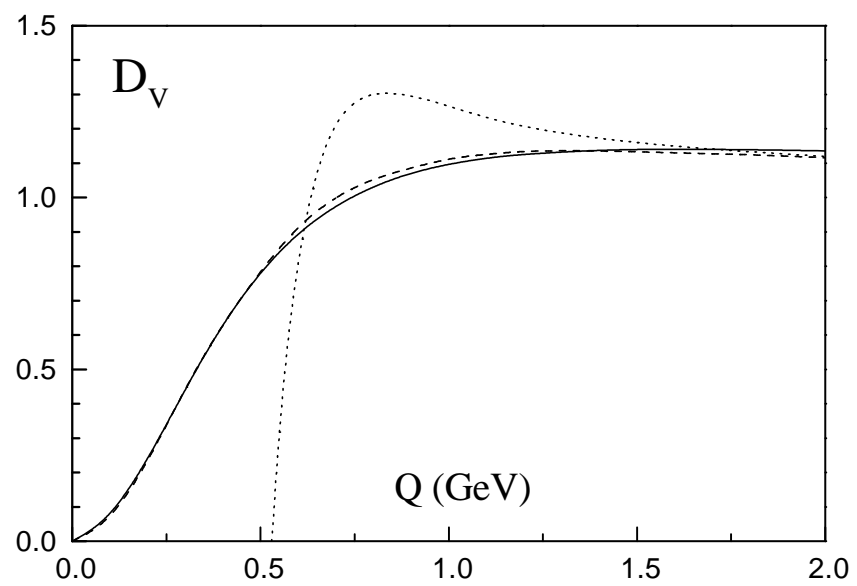

FIG. 1. The light Adler function corresponding to the non-strange vector channel of $\tau$ decay data. The solid curve is the APT result. The experimental curve (dashed line) corresponding to the ALEPH data and the perturbative result with power corrections (dotted line) are taken from Ref. [11]. 